\title{
A Severe Sight-threatening Complication of Herpes Zoster Ophthalmicus: Orbital Apex Syndrome
}

\author{
Sumru Onal1,2, Günay Uludağ³, Mustafa Mert Metin, Aslıhan Onay5, Önder Ergönul6 \\ 1 Department of Ophthalmology, Koç University School of Medicine, İstanbul, Turkey \\ 2 Department of Ophthalmology, V.K.V. American Hospital, İstanbul, Turkey \\ 3 Department of Ophthalmology, Koç University Hospital, İstanbul, Turkey \\ 4 Koç University School of Medicine, İstanbul, Turkey \\ 5 Department of Radiology, Koç University School of Medicine, İstanbul, Turkey \\ 6 Department of Infectious Diseases and Clinical Microbiology, Koç University School of Medicine, İstanbul, Turkey
}

\begin{abstract}
A 39-year old male patient presented with severe herpes zoster ophthalmicus (HZO) on the left side of his face and developed orbital apex syndrome (OAS) despite systemic valacyclovir therapy. Persistent low vision, ptosis, limitation of extraocular muscle movements, anisocoria, epiphora, and photophobia led to suspicion of OAS. OAS was confirmed by orbital MRI. Intravenous acyclovir and systemic corticosteroids were initiated. Gradual recovery occurred over six months. The patient was seronegative for HIV and syphilis. He reported alcohol consumption for the last few months. This case increased awareness of a rare, but a sight-threatening complication of HZO in an immunocompetent patient.

Keywords: Herpes zoster ophthalmicus; Orbital apex syndrome; Immunocompetence; Acyclovir; Optic Nerve
\end{abstract}

\section{INTRODUCTION}

$\mathrm{V}$ aricella-zoster virus (VZV) is found in worldwide distribution and can lead to two distinct viral syndromes. Primary infection occurs as varicella and is a contagious and usually benign childhood disease (1). Herpes zoster (HZ) presents as unilateral pain in a dermatomal distribution accompanied by a vesicular rash. Herpes zoster generally occurs in older adults and results from the reactivation of latent VZV within sensory spinal or cerebral ganglia (2).

Herpes zoster ophthalmicus (HZO) occurs as a result of $\mathrm{HZ}$ involvement of the ophthalmic division of the trigeminal nerve. Involvement of the nasociliary branch of the ophthalmic nerve leads to Hutchinson sign which is characterized by the occurrence of skin lesions at the tip, side, or root of the nose. Hutchinson sign is accepted as a strong predictor of ocular inflammation and corneal denervation in HZO. Herpes zoster ophthalmicus accounts for 10 to $20 \%$ of cases of HZ, and therefore, every indi-
Corresponding Author:

Sumru Onal

E-mail:

sumruo_md@yahoo.com

Received: December 06, 2019

Accepted: December 11, 2019

Published: December 25, 2019

\section{Suggested citation:}

Onal S, Uludağ G, Metin MM, Onay A, Önder E. A severe sight-threatening complication of herpes zoster ophthalmicus: Orbital Apex Syndrome. Infect Dis Clin Microbiol 2019; 3: 143 148.

DOI: 10.36519/idcm.2019.19029 


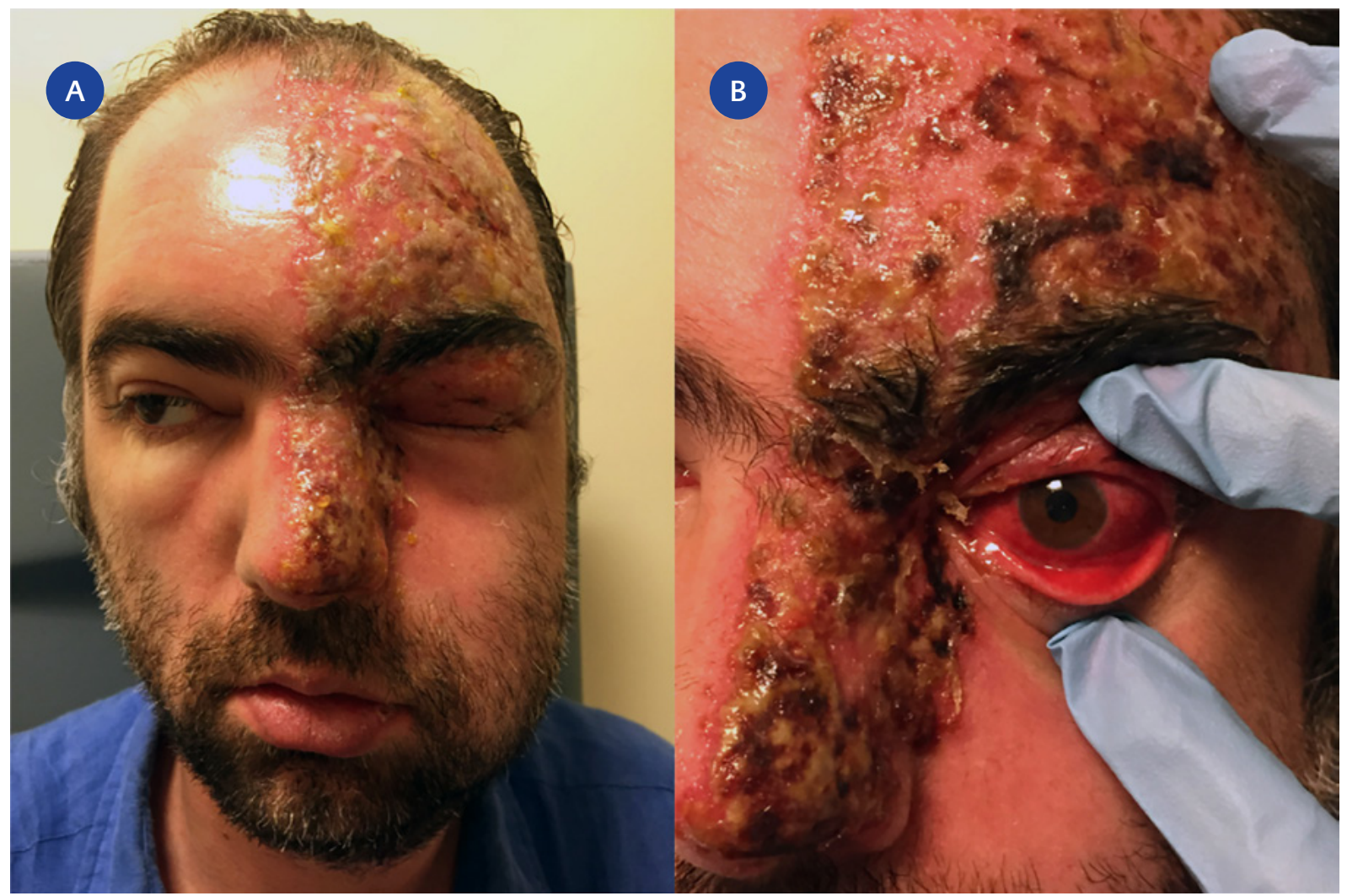

Figure 1. Facial photograph of the patient at presentation shows severe facial edema and drooping and vesiculopustular lesions on frontal and scalp skin, upper eyelid, medial canthus, and root, side and tip of nose on the left side of his face (A). Lesions do not cross the midline. Crusting of the vesicular skin lesions and severe diffuse anterior scleritis is seen in the left eye 2 days after treatment (B).

\section{HIGHLIGHTS}

- The severe course of HZO infection might be related to the delay in initiation of systemic antiviral therapy and inadvertent use of corticosteroids.

- Orbital apex syndrome should be suspected in patients with HZO who develop signs and symptoms associated with the dysfunction of fifth, third, fourth, sixth cranial nerves, and in particular the optic nerve.

- Orbital apex syndrome secondary to HZO may develop despite oral antiviral therapy in immunocompetent individuals.

- Intravenous acyclovir and corticosteroids are warranted in the immediate treatment of OAS, which is a severe and vision-threatening complication associated with HZO. vidual has about $1 \%$ risk of developing $\mathrm{HZO}$ during lifetime (2).

We herein present an immunocompetent male patient with severe $\mathrm{HZO}$ complicated by orbital apex syndrome (OAS), which is a rare and potentially blinding complication of $\mathrm{HZO}$.

\section{CASE PRESENTATION}

A 39-year old male patient presented in August 2015 to the Ophthalmology Department with severe facial and eyelid oedema and facial drooping on the left side of his face. An accompanying vesiculopustular rash respecting facial midline was observed on frontal and scalp skin, upper eyelid, medial canthus, and root, side and tip of the nose on the left side of his face (Figure 1A). He was complaining of worsening left-sided headache for eight days and eyelid oedema that started six days ago. He had been given corticosteroid ointment for the 


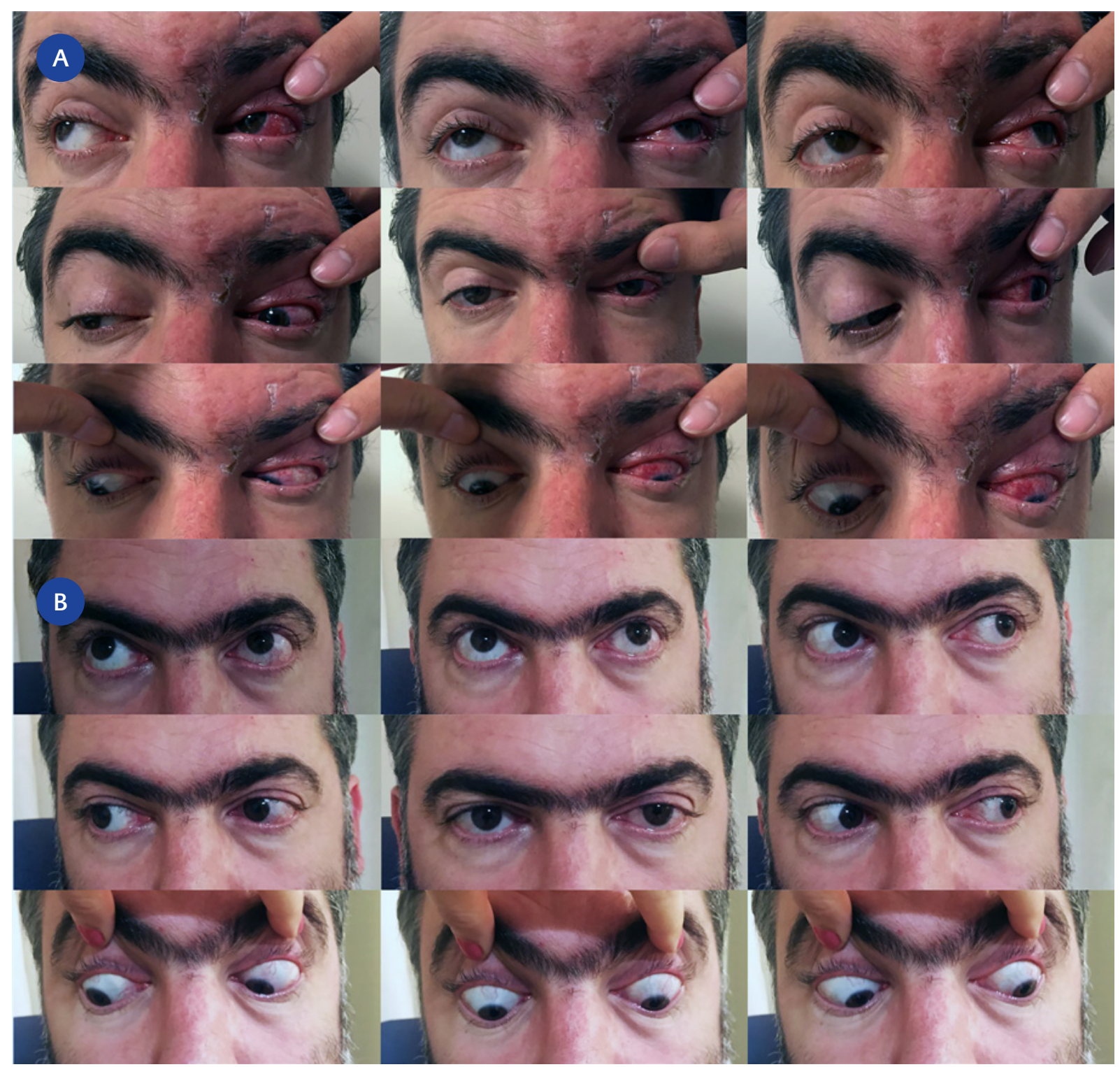

Figure 2. There is limitation in extraocular muscle movements in 6 cardinal directions of gaze and upward and downward gaze in the left eye. The left upper eyelid is being elevated as there is ptosis of the left upper eyelid. Recovery of skin lesions and persistence of scleral inflammation is evident 12 days after oral antiviral treatment began (A). Six months after presentation there is complete recovery in ptosis and in extraocular muscle movements in all directions of gaze in the left eye (B).

diagnosis of contact dermatitis elsewhere. The patient reported moderate alcohol consumption for the last few months; otherwise, his medical and family history was unremarkable.

Ophthalmological examination revealed a visual acuity of $1.0 \mathrm{OD}$ and 0.3 , with the pinhole, OS. Biomicroscopy showed severe eyelid oedema, vesiculopustular lesions and madarosis in the left upper eyelid, and vesiculopustular lesions in the medial canthus area skin. Diffuse scleritis, ciliary injection, interstitial keratitis with diffuse corneal stromal edema, medium-sized keratic precipitates, and 2.5 $(+)$ cells in the anterior chamber were observed in the left eye. Biomicroscopy of the right eye was within normal limits. Intraocular pressures were $14 \mathrm{mmHg} O D$ and $10 \mathrm{mmHg}$ OS. The fundus of the right eye was normal, despite limited view, the posterior pole and peripheral retina were normal in the left eye. The patient was diagnosed with $\mathrm{HZO}$ and 


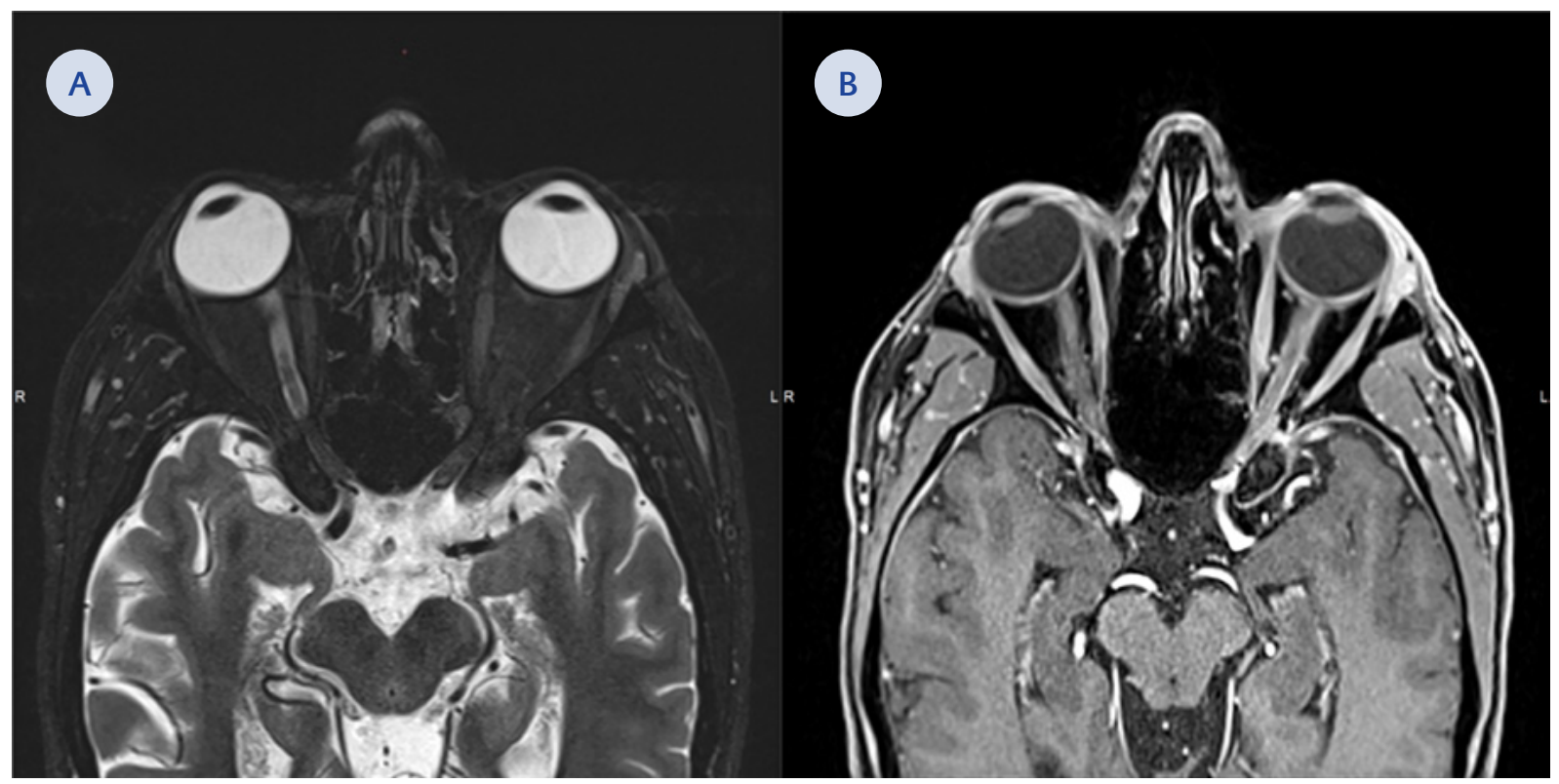

Figure 3. Axial magnetic resonance images of the patient taken 12 days after presentation. T2 weighted image shows swelling and edema of the extraocular muscles and optic nerve on the left side. There is no cerebrospinal fluid visible along the optic nerve tract on the left side when compared to the optic nerve tract on the right side (A). Post-contrast T1 weighted fat suppressed image shows enhancement of the extraocular muscles and the optic nerve sheath especially near the orbital apex. A subtle contrast enhancement is also seen within the bony orbital apex (B).

was started on oral valacyclovir $3 \mathrm{~g} /$ day and amoxicillin-clavulanate $2 \mathrm{~g} /$ day. The topical treatment prescribed for the left eye consisted of ganciclovir 0.15\% ophthalmic gel five times per day, topical prednisolone acetate 1\% tid, and tropicamide 1\% tid. Figure 1B shows crusting vesicular lesions in the distribution of ophthalmic division of trigeminal nerve, with a positive Hutchinson sign on the left side and severe diffuse anterior scleritis in the left eye two days after treatment was initiated.

Twelve days after presentation, visual acuity remained at 0.7 in the left eye despite regression of keratitis and anterior uveitis and in spite of oral valacyclovir and topical treatment. Because of the persistence of left-sided ptosis despite the recovery of skin and eyelid lesions, limitation in extraocular muscle movements leading to intermittent diplopia upon elevation of the upper eyelid, and epiphora and photophobia when exposed to ambient light cranial and orbital magnetic resonance imaging (MRI) was ordered. The patient was consulted with the Infectious Diseases Department. Figure 2A shows limitation in extraocular muscle movements in all directions of gaze and persistence of diffuse scleritis in the left eye. The patient also had anisocoria, with a left-sided mid-dilated pupil that was unresponsive to light and accommodation. Orbital MRI revealed enhancement and swelling of the left extraocular muscles, enhancement of the left optic nerve sheath, with cerebrospinal fluid invisible along the optic nerve on the left side (Figure 3). The patient was diagnosed with OAS secondary to HZO. Cranial MRI was unrewarding. Serological tests for RPR, TPHA, and anti-HIV were negative. Upon diagnosis of orbital apex syndrome, the patient was switched to intravenous acyclovir $3 \mathrm{~g} /$ day. Oral methylprednisolone $64 \mathrm{mg} /$ day $(0.8 \mathrm{mg} / \mathrm{kg} /$ day) was added to his treatment 24 hours after initiation of intravenous acyclovir. Intravenous acyclovir treatment was given for 15 days, and oral corticosteroids were slowly tapered off over 12 weeks. After completion of intravenous antiviral therapy, the patient was started on oral valacyclovir $3 \mathrm{~g} /$ day until oral corticosteroids were discontinued. After that, valacyclovir dose was decreased to $500 \mathrm{mg} /$ day.

Left-sided ptosis and limitation of extraocular muscle movements gradually recovered over six months (Figure 2B). Oral valacyclovir was discontin- 
ued at his final visit in March 2016. Visual acuity of the left eye improved to 1.0. The patient developed faint patchy iris stromal atrophy in the left eye and had two episodes of recurrent anterior uveitis that were treated with topical corticosteroids. Pupillary reactions returned to normal; however, the pupil remained mildly distorted due to pupillary sphincter involvement.

\section{DISCUSSION AND CONCLUSION}

The case reported herein represents HZO complicated by OAS in a patient with full-blown ocular affection, presenting with scleral, corneal, and iris involvement. Orbital apex syndrome is an infrequent but severe and potentially blinding complication of HZO. Herpes zoster ophthalmicus complicated by OAS, which is also known as optic neuritis/neuropathy associated with complete ophthalmoplegia, was first reported by Ramsell in 1967 (3).

To our knowledge there are only limited number of case reports in the literature on the occurrence of OAS as a complication of HZO $(3,13)$. Orbital apex syndrome may result from inflammatory, infectious, neoplastic, iatrogenic, traumatic, or vascular conditions affecting the orbital apex region. It is characterized by dysfunction of ophthalmic, oculomotor, trochlear, abducens nerves and particularly the optic nerve (14). It has been hypothesized that OAS associated with $\mathrm{HZO}$ can be secondary to compression caused by intraorbital inflammation or myositis. A direct viral cytopathic effect caused by viral dissemination from the HZ virus infecting the fifth cranial nerve to the neighbouring cranial nerves in the orbital apex has also been proposed. An immunologically mediated response to the virus might also induce perineural inflammation, peripheral nerve demyelination, contiguous orbital inflammation, cranial vasculitis, myositis, brainstem encephalitis and meningitis $(6,8)$. We did not observe any symptoms or signs, as confirmed by cranial MRI imaging, that would suggest intracranial involvement in our patient.

The severe course of HZO infection in our patient might be related to the delay in initiation of systemic antiviral therapy, inadvertent use of corticosteroid ointment before the presentation, and moderate alcohol consumption defined as up to two drinks per day for men (15). Otherwise, the patient was immunocompetent.

Orbital apex syndrome should be suspected in patients with $\mathrm{HZO}$ who develop signs and symptoms associated with the dysfunction of fifth, third, fourth, sixth cranial nerves, and in particular the optic nerve. Orbital apex syndrome secondary to HZO may develop despite oral antiviral therapy in immunocompetent individuals. Intravenous acyclovir and corticosteroids are warranted in the immediate treatment of OAS, which is a severe and vision-threatening complication associated with $\mathrm{HZO}$.
Informed Consent: Written consent was obtained from the patient.

Peer-review: Externally peer-reviewed

Author Contributions: Concept - S.Ö., Ö.E.; Design - S.Ö., G.U., M.M.M., A.O., Ö.E.; Supervision - S.Ö., Ö.E.; Materials - M.M.M., A.O., G.U.; Data Collection and/or Processing - G.U., A.O., M.M.M.; Analysis and/or Interpretation - S.Ö., G.U., A.O., M.M.M., Ö.E.;
Literature Review - M.M.M., A.O., G.U.; Writer - S.Ö.; Critical Reviews - Ö.E.

Conflict of Interest: The authors have no conflict of interest to declare.

Financial Disclosure: The authors declared that this study has received no financial support. 


\section{REFERENCES}

1 Arvin AM. Varicella-zoster virus. Clin Microbiol Rev 1996; 9: 361-81.

2 Liesegang TJ. Herpes zoster ophthalmicus natural history, risk factors, clinical presentation, and morbidity. Ophthalmology 2008; 115: 3-12.

3 Ramsell TG. Complications of herpes zoster ophthalmicus. Am J Ophthalmol 1967; 63:1796-8.

4 Verhaeghe F, Villain M, Labauge P, Daien V. Orbital Apex Syndrome Secondary to Herpes Zoster Ophthalmicus. J Neuroophthalmol 2016; 36:147-51.

5 Lee CY, Tsai HC, Lee SS, Chen YS. Orbital apex syndrome: an unusual complication of herpes zoster ophthalmicus. BMC Infect Dis 2015; 15: 33.

6 Merino-Iglesias A, Montero JA, Calabuig-Goena M, Giral do-Agudelo LF. Orbital apex syndrome secondary to herpes zoster virus infection. BMJ Case Rep 2014; 2014.

7 Arda H, Mirza E, Gumus K, Oner A, Karakucuk S, Sirakaya E. Orbital apex syndrome in herpes zoster ophthalmicus. Case Rep Ophthalmol Med 2012; 2012: 854503.

8 Kurimoto T, Tonari M, Ishizaki N, Monta M, Hirata S, Oku H, et al. Orbital apex syndrome associated with herpes zoster ophthalmicus. Clin Ophthalmol 2011; 5: 1603-8.
9 Saxena R, Phuljhele S, Aalok L, Sinha A, Menon V, Sharma P, et al. A rare case of orbital apex syndrome with herpes zoster ophthalmicus in a human immunodeficiency virus-positive patient. Indian J Ophthalmol 2010; 58: 527-30.

10 Dhingra S, Williams G, Pearson A. Severe, permanent orbital disease in herpes zoster ophthalmicus. Orbit 2008; 27: 325-7.

11 Shirato S, Oshitari T, Hanawa K, Adachi-Usami E. Magnetic resonance imaging in case of cortical apex syndrome caused by varicella zoster virus. Open Ophthalmol J 2008; 2: 109-11.

12 Bourke RD, Pyle J. Herpes zoster ophthalmicus and the orbital apex syndrome. Aust N Z J Ophthalmol 1994; 22: 77-80.

13 Kattah JC, Kennerdell JS. Orbital apex syndrome secondary to herpes zoster ophthalmicus. Am J Ophthalmol 1978; 85: 37882.

14 Yeh S, Foroozan R. Orbital apex syndrome. Curr Opin Ophthalmol 2004; 15: 490-498.

15 US Department of Health and Human Services and US Department of Agriculture. 2015-2020 Dietary Guidelines for Americans. 8th ed. Washington, DC: US Dept of Health and Human Services and US Dept of Agriculture; 2015. 